\title{
RICCI TENSORS OF REAL HYPERSURFACES IN A COMPLEX PROJECTIVE SPACE
}

\author{
SADAHIRO MAEDA \\ (Communicated by Christopher Croke) \\ Dedicated to Professor Saburo Kitamura on his 65th birthday
}

\begin{abstract}
This paper gives a classification of real hypersurfaces in a complex projective space under assumptions that the structure vector $\xi$ is principal, the focal map has constant rank, and $\nabla_{\xi} S=0$, where $S$ is the Ricci tensor of the real hypersurface.
\end{abstract}

\section{INTRODUCTION}

Let $P_{n}(C)$ be an $n$-dimensional complex projective space with Fubini-Study metric $G$ of constant holomorphic sectional curvature 4 , and let $M$ be a real hypersurface of $P_{n}(C)$. Then $M$ has an almost contact metric structure $(\phi, \xi, \eta, g)$ (cf. $\S 1$ ) induced from the complex structure $J$ of $P_{n}(C)$. Many differential geometers have studied $M$ by using structure $(\phi, \xi, \eta, g)$. Typical examples of real hypersurfaces in $P_{n}(C)$ are homogeneous ones. Takagi [9] classified homogeneous real hypersurfaces in $P_{n}(C)$. By virtue of his work, we find that a homogeneous real hypersurface of $P_{n}(C)$ is locally congruent to one of the six model spaces of type $A_{1}, A_{2}, B, C, D$, and $E$ (see Theorem A).

It is well known that there exist no Einstein real hypersurfaces $M$ in $P_{n}(C)$, $n \geq 3$ (cf. [5]). Moreover, $P_{n}(C) \quad(n \geq 3)$ does not admit real hypersurfaces $M$ with parallel Ricci tensor $S$ (that is, $\nabla_{X} S=0$ for each vector field $X$ tangent to $M$, where $\nabla$ denotes the induced Riemannian connection from the Riemmanian connection $\tilde{\nabla}$ of $P_{n}(C)$; cf. [2]). So, it is natural to investigate real hypersurfaces $M$ by using some conditions (on the derivative of $S$ ) which are weaker than $\nabla S=0$.

The purpose of this paper is to classify real hypersurfaces $M$ in $P_{n}(C)$ satisfying $\nabla_{\xi} S=0$ (that is, the Ricci tensor $S$ is parallel in the direction of the structure vector $\xi=-J N$, where $N$ is a unit normal vector field on $M$ ) under the condition that $\xi$ is a principal curvature vector of $M$ and that the focal map has constant rank on $M$. We have

Received by the editors December 23, 1992 and, in revised form, March 30, 1993.

1991 Mathematics Subject Classification. Primary 53C40.

Key words and phrases. Real hypersurfaces, principal curvature vectors, Ricci tensors, tubes.

This research was partially supported by Grant-in-Aid for Scientific Research (No. 04640050). Ministry of Education, Science and Culture. 
Theorem. Let $M$ be a real hypersurface of $P_{n}(C) \quad(n \geq 3)$ on which $\xi$ is a principal curvature vector with principal curvature $\alpha=2 \cot 2 r$ and the focal map $\phi_{r}$ has constant rank on $M$. If $\nabla_{\xi} S=0$, then $M$ is locally congruent to one of the following:

(1) a homogeneous real hypersurface which lies on a tube of radius $r$ over a totally geodesic $P_{k}(C) \quad(1 \leq k \leq n-1)$, where $0<r<\pi / 2$;

(2) a homogeneous real hypersurface which lies on a tube of radius $r$ over a complex quadric $Q_{n-1}$, where $0<r<\pi / 4$ and $\cot ^{2} 2 r=n-2$;

(3) a homogeneous real hypersurface which lies on a tube of radius $r$ over $P_{1}(C) \times P_{(n-1) / 2}(C)$, where $0<r<\pi / 4, \cot ^{2} 2 r=1 /(n-2)$, and $n(\geq 5)$ is odd;

(4) a homogeneous real hypersurface which lies on a tube of radius $r$ over a complex Grassmann $G_{2,5}(C)$, where $0<r<\pi / 4, \cot ^{2} 2 r=3 / 5$, and $n=9$;

(5) a homogeneous real hypersurface which lies on a tube of radius $r$ over a Hermitian symmetric space $\mathrm{SO}(10) / \mathrm{U}(5)$, where $0<r<\pi / 4, \cot ^{2} 2 r=5 / 9$, and $n=15$;

(6) a nonhomogeneous real hypersurface which lies on a tube of radius $\pi / 4$ over a Kaehler submanifold $\widetilde{N}$ with nonzero principal curvatures $\neq \pm 1$;

(7) a nonhomogeneous real hypersurface which lies on a tube of radius $r$ over a k-dimensional Kaehler submanifold $\widetilde{N}$ on which the rank of each shape operator is not greater than 2 with nonzero principal curvatures $\neq$ $\pm((2 k-1) /(2 n-2 k-1))^{1 / 2}$ and $\cot ^{2} r=(2 k-1) /(2 n-2 k-1)$, where $k=1, \ldots, n-1$.

\section{Preliminaries}

Let $M$ be an orientable real hypersurface of $P_{n}(C)$, and let $N$ be a unit normal vector field on $M$. The Riemannian connections $\tilde{\nabla}$ in $P_{n}(C)$ and $\nabla$ in $M$ are related by the following formulas for any vector fields $X$ and $Y$ on $M$ :

$$
\begin{gathered}
\tilde{\nabla}_{X} Y=\nabla_{X} Y+g(A X, Y) N, \\
\tilde{\nabla}_{X} N=-A X,
\end{gathered}
$$

where $g$ denotes the Riemannian metric of $M$ induced from the Fubini-Study metric $G$ of $P_{n}(C)$ and $A$ is the shape operator of $M$ in $P_{n}(C)$. An eigenvector $X$ of the shape operator $A$ is called a principal curvature vector. Also an eigenvalue $\lambda$ of $A$ is called a principal curvature. In what follows, we denote by $V_{\lambda}$ the eigenspace of $A$ associated with eigenvalue $\lambda$. It is known that $M$ has an almost contact metric structure induced from the complex structure $J$ on $P_{n}(C)$; that is, we define a tensor field $\phi$ of type (1.1), a vector field $\xi$, and a 1form $\eta$ on $M$ by $g(\phi X, Y)=G(J X, Y)$ and $g(\xi, X)=\eta(X)=G(J X, N)$. Then we have

$$
\phi^{2} X=-X+\eta(X) \xi, \quad g(\xi, \xi)=1, \quad \phi \xi=0 .
$$

It follows from (1.1) that

$$
\begin{gathered}
\left(\nabla_{X} \phi\right) Y=\eta(Y) A X-g(A X, Y) \xi, \\
\nabla_{X} \xi=\phi A X .
\end{gathered}
$$


Let $\widetilde{R}$ and $R$ be the curvature tensors of $P_{n}(C)$ and $M$, respectively. Since the curvature tensor $\widetilde{R}$ has a nice form, we have the following Gauss and Codazzi equations:

$$
\begin{aligned}
& g(R(X, Y) Z, W)=g(Y, Z) g(X, W)-g(X, Z) g(Y, W) \\
& +g(\phi Y, Z) g(\phi X, W) \\
& -g(\phi X, Z) g(\phi Y, W)-2 g(\phi X, Y) g(\phi Z, W) \\
& +g(A Y, Z) g(A X, W)-g(A X, Z) g(A Y, W) \text {, } \\
& \text { From (1.3), (1.5)-(1.7) we get }
\end{aligned}
$$

$$
\begin{aligned}
S X= & (2 n+1) X-3 \eta(X) \xi+h A X-A^{2} X, \\
\left(\nabla_{X} S\right) Y= & -3\{g(\phi A X, Y) \xi+\eta(Y) \phi A X\}+(X h) A Y \\
& +(h I-A)\left(\nabla_{X} A\right) Y-\left(\nabla_{X} A\right) A Y,
\end{aligned}
$$

where $h=\operatorname{trace} A, S$ is the Ricci tensor of type $(1,1)$ on $M$, and $I$ is the identity map.

In the following, we use the same terminology and notation as above unless otherwise stated. Now we prepare the following without proof in order to prove our Theorem:

Theorem A [9]. Let $M$ be a homogeneous real hypersurface of $P_{n}(C)$. Then $M$ is a tube of radius $r$ over one of the following Kaehler submanifolds:

$\left(\mathrm{A}_{1}\right)$ hyperplane $P_{n-1}(C)$, where $0<r<\pi / 2$;

$\left(\mathrm{A}_{2}\right)$ totally geodesic $P_{k}(C) \quad(1 \leq k \leq n-2)$, where $0<r<\pi / 2$;

(B) complex quadric $Q_{n-1}$, where $0<r<\pi / 4$;

(C) $P_{1}(C) \times P_{(n-1) / 2}(C)$, where $0<r<\pi / 4$ and $n(\geq 5)$ is odd;

(D) complex Grassmann $G_{2,5}(C)$, where $0<r<\pi / 4$ and $n=9$; 15 .

(E) Hermitian symmetric space $\mathrm{SO}(10) / \mathrm{U}(5)$, where $0<r<\pi / 4$ and $n=$

Theorem B [3]. Let $M$ be a real hypersurface of $P_{n}(C)$. Then $M$ has constant principal curvatures and $\xi$ is a principal curvature vector if and only if $M$ is locally congruent to a homogeneous real hypersurface.

Theorem C [4]. Let $M$ be a real hypersurface of $P_{n}(C)$. If $\nabla_{\xi} A=0$, then $\xi$ is a principal curvature vector; in addition, except for the null set on which the focal map $\phi_{r}$ degenerates, $M$ is locally congruent to one of the following.

(i) a homogeneous real hypersurface which lies on a tube of radius $r$ over a totally geodesic $P_{k}(C) \quad(1 \leq k \leq n-1)$, where $0<r<\pi / 2$;

(ii) a nonhomogeneous real hypersurface which lies on a tube of radius $\pi / 4$ over a Kaehler submanifold $\tilde{N}$ with nonzero principal curvatures $\neq \pm 1$.

Proposition A [6]. If $\xi$ is a principal curvature vector, then the corresponding principal curvature $\alpha$ is locally constant.

Proposition B [6]. Assume that $\xi$ is a principal curvature vector and the corresponding principal curvature is $\alpha$. If $A X=\lambda X$ for $X \perp \xi$, then we have $A \phi X=((\alpha \lambda+2) /(2 \lambda-\alpha)) \phi X$. 
Proposition C [1]. Let $M$ be a connected orientable real hypersurface (with unit normal vector $N)$ in $P_{n}(C)$ on which $\xi$ is a principal curvature vector with principal curvature $\alpha=2 \cot 2 r$ and the focal map $\phi_{r}$ has constant rank on $M$. Then the following hold:

(i) $M$ lies on a tube (in the direction of $\eta=\gamma^{\prime}(r)$, where $\gamma(r)=\exp _{x}(r N)$ and $x$ is a base point of the normal vector $N$ ) of radius $r$ over a certain Kaehler submanifold $\tilde{N}$ in $P_{n}(C)$.

(ii) Let $\cot \theta$ be a principal curvature of the shape operator $A_{\eta}$ at $y=\gamma(r)$ of the Kaehler submanifold $\widetilde{N}$. Then the real hypersurface $M$ has a principal curvature $\cot (r-\theta)$ at $x=\gamma(0)$.

Finally we prepare the following three lemmas.

Lemma 1. If $A \xi=0$, then $\nabla_{\xi} A=0$.

Proof. Since $\xi$ is a principal curvature vector, in general (1.5) and Proposition A assert that

$$
\left(\nabla_{\xi} A\right) \xi=0 .
$$

Let $X$ be a principal curvature (unit) vector orthogonal to $\xi$ with principal curvature $\lambda$. Then Proposition B shows that

$$
A \phi X=(1 / \lambda) \phi X .
$$

Now, for any principal curvature vector $X$ orthogonal to $\xi$ we have

$$
\begin{aligned}
\left(\nabla_{\xi} A\right) X & =\left(\nabla_{X} A\right) \xi+\phi X \quad(\text { from }(1.7) \\
& \left.=\nabla_{X}(A \xi)-A \nabla_{X} \xi+\phi X=-A \phi A X+\phi X \quad \text { (from } A \xi=0 \text { and }(1.5)\right) \\
& =0 \quad(\text { from }(1.11)) .
\end{aligned}
$$

So, we conclude that $\nabla_{\xi} A=0$.

Lemma 2. If $\xi$ is a principal curvature vector, then $\xi(\operatorname{trace} A)=0$.

Proof. For each principal curvature (unit) vector $X$ orthogonal to $\xi$ with principal curvature $\lambda$, from (1.5), (1.7) and Propositions A and B we get

$$
\left(\nabla_{\xi} A\right) X=\alpha\{\lambda-(\alpha \lambda+2) /(2 \lambda-\alpha)\} / 2 \cdot \phi X .
$$

Hence $(1.10)$ and $(1.12)$ imply $\operatorname{trace}\left(\nabla_{\xi} A\right)=0$, so $\xi(\operatorname{trace} A)\left(=\operatorname{trace}\left(\nabla_{\xi} A\right)\right)=$ 0 .

Lemma 3. If $\nabla_{\xi} A=0$, then $\nabla_{\xi} S=0$.

Proof. We note that $\nabla_{\xi} A=0$ implies $\xi$ is a principal curvature vector (cf. Theorem $\mathrm{C}$ ) and $\xi(\operatorname{trace} A)=0$. Then (1.9) tells us that $\nabla_{\xi} A=0$ implies $\nabla_{\xi} S=0$.

\section{Proof of Theorem}

By hypothesis we may put $A \xi=\alpha \xi$. From Proposition A our discussion is divided into two cases: (i) $\alpha=0$ and (ii) $\alpha \neq 0$.

Case (i): $\alpha=0$. First we remark that our real hypersurface $M$ satisfies $\nabla_{\xi} A=0$ (cf. Lemma 1). Hence by virtue of Theorem $\mathrm{C}$ we find that $M$ is locally congruent to a homogeneous real hypersurface which lies on a tube 
of radius $\pi / 4$ over a totally geodesic $P_{k}(C)(1 \leq k \leq n-1)$ or congruent to a nonhomogeneous real hypersurface which lies on a tube of radius $\pi / 4$ over a Kaehler submanifold $\tilde{N}$ with nonzero principal curvatures $\neq \pm 1$, that is, $M$ is of case (1) with $r=\pi / 4$ or of case (6) in Theorem. Of course, these examples satsify $\nabla_{\xi} S=0$ (see Lemma 3 ).

Case (ii): $\alpha \neq 0$. In the following, $X$ denotes a principal curvature (unit) vector orthogonal to $\xi$ with principal curvature $\lambda$. Since $\xi$ is a principal curvature vector, $\left(\nabla_{\xi} S\right) \xi=0$ holds (see, (1.9), (1.10), and Lemma 2). So we have only to determine real hypersurfaces $M$ satisfying $\left(\nabla_{\xi} S\right) X=0$ for any $X$. In case $\lambda=(\alpha \lambda+2) /(2 \lambda-\alpha)$, from (1.9), (1.12), and Lemma 2 we find that $\left(\nabla_{\xi} S\right) X=0$. Next we consider the case of $\lambda \neq(\alpha \lambda+2) /(2 \lambda-\alpha)$. Here, for simplicity we put $a=\alpha\{\lambda-(\alpha \lambda+2) / 2 \lambda-\alpha)\} / 2$. Note that $a \neq 0$. It follows from (1.9), (1.12), and Lemma 2 that

$$
\left(\nabla_{\xi} S\right) X=a\{h-\lambda-(\alpha \lambda+2) /(2 \lambda-\alpha)\} \phi X .
$$

Hence $\lambda$ must satisfy the following quadratic equation for $\lambda$ :

$$
h-\lambda-(\alpha \lambda+2) /(2 \lambda-\alpha)=0 \text {. }
$$

Since $\xi$ is a principal curvature vector and the focal map $\phi_{r}$ has constant rank on $M$, our manifold $M$ is a tube (of radius $r$ ) over a certain ( $k$-dimensional) Kaehler submanifold $\tilde{N}$ in $P_{n}(C)$. So we may put $\alpha=2 \cot 2 r(=\cot r-\tan r)$ (cf. Proposition C). Hence, solving the equation $\lambda-(\alpha \lambda+2) /(2 \lambda-\alpha)=0$, we see that $\lambda=\cot r,-\tan r$. We here denote by $\lambda_{1}, \lambda_{2}(\neq \cot r,-\tan r)$ the solutions for (1.13). Note that (cf. Proposition B)

$$
\phi V_{\cot r}=V_{\cot r}, \quad \phi V_{-\tan r}=V_{-\tan r}, \quad \text { and } \phi V_{\lambda_{1}}=V_{\lambda_{2}} .
$$

Then $M$ has at most five distinct principal curvatures $2 \cot 2 r$ (with multiplicity 1 ), $\cot r$ (with multiplicity $2 n-2 k-2$ ), $-\tan r$ (with multiplicity $2 k-2 m$ ), $\lambda_{1}$ (with multiplicity $m \geq 0$ ), and $\lambda_{2}$ (with multiplicity $m \geq 0$ ). We here note that the multiplicity of the principal curvature $\cot r$ is determined by the result of Proposition 3.1 of [1]. Hence

$$
h=(2 n-2 k-1) \cot r-(2 k-2 m+1) \tan r+m\left(\lambda_{1}+\lambda_{2}\right) .
$$

On the other hand,

$$
h=\lambda_{1}+\lambda_{2} \text {. }
$$

It follows from (1.15) and (1.16) and $\lambda_{2}=\left(\alpha \lambda_{1}+2\right) /\left(2 \lambda_{1}-\alpha\right)$ that

$$
\begin{aligned}
& (2 n-2 k-1) \cot r-(2 k-2 m+1) \tan r \\
& \quad+(m-1)\left\{\lambda_{1}+\left(\alpha \lambda_{1}+2\right) /\left(2 \lambda_{1}-\alpha\right)\right\}=0 .
\end{aligned}
$$

In the following, Case (ii) is divided into three cases: (I) $m=0$, (II) $m=1$, and (III) $m \geq 2$.

Case (I). Our manifold $M$ is of case (1) with $0<r(\neq \pi / 4)<\pi / 2$ in Theorem (cf. [6]). As a matter of course, the manifold $M$ satisfies $\nabla_{\xi} S=0$ (cf. Theorem $\mathrm{C}$ and Lemma 3).

Case (II). Our real hypersurface $M$ has at most five distinct principal curvatures $2 \cot 2 r$ (with multiplicity 1 ) $\cot r$ (with multiplicity $2 n-2 k-2$ ), $-\tan r$ 
(with multiplicity $2 k-2$ ), $\lambda_{1}$ (with multiplicity 1 ), and $\lambda_{2}$ (with multiplicity 1). Since the multiplicities of the principal curvatures of $M$ do not match the multiplicities of any homogeneous real hypersurface, the manifold $M$ is not homogeneous (cf. [10]). Hence, both $\lambda_{1}$ and $\lambda_{2}$ are not constant (cf. Theorem B). Moreover, Proposition $C$ shows that $\lambda_{1}$ and $\lambda_{2}$ can, respectively, be expressed as $\lambda_{1}=\cot (r-\theta)$ and $\lambda_{2}=\cot (r+\theta)$. where $\cot \theta$ is a principal curvature of the Kaehler submanifold $\tilde{N}$. In addition, Equation (1.17) yields

$$
\cot ^{2} r=(2 k-1) /(2 n-2 k-1) \text {. }
$$

Hence the manifold $M$ satisfying (1.14), (1.16), and (1.18) is of case (7) in Theorem.

Case (III). It follows from (1.17) and Proposition A that $\lambda_{1}$ is constant. Therefore, we can see that our manifold $M$ is homogeneous (cf. Theorem B). Now we shall check $\nabla_{\xi} S=0$ one-by-one for homogeneous real hypersurfaces of types (B), (C), (D), and (E).

Let $M$ be of type (B) (which is a tube of radius $r$ ). Let $x=\cot r$. Then $M$ has three distinct constant principal curvatures $r_{1}=(1+x) /(1-x)$ with multiplicity $n-1, r_{2}=(x-1) /(x+1)$ with multiplicity $n-1$, and $\alpha=$ $\left(x^{2}-1\right) / x$ with multiplicity 1 (cf. [10]). Since $\phi V_{r_{1}}=V_{r_{2}}, \nabla_{\xi} S=0$ is equivalent to $h-r_{1}-r_{2}=0$. Then we have the equation $x^{4}-2(2 n-3) x^{2}+1=0$. Hence, we find $x^{2}=2 n-3 \pm 2 \sqrt{(n-1)(n-2)}$ so that $x=\sqrt{n-1}+\sqrt{n-2}$, since $x>1$. So $M$ is of case (2) in Theorem. Now let $M$ be of type (C) (which is a tube of radius $r$ ). Let $x=\cot r$. Then $M$ has five distinct constant principal curvatures $r_{1}=(1+x) /(1-x)$ with multiplicity $2, r_{2}=(x-1) /(x+1)$ with multiplicity $2, r_{3}=x$ with multiplicity $n-3, r_{4}=-1 / x$ with multiplicity $n-3$, and $\alpha=\left(x^{2}-1\right) / x$ with multiplicity 1 (cf. [10]). Since $\phi V_{r_{1}}=V_{r_{2}}, \phi V_{r_{3}}=V_{r_{3}}$, and $\phi V_{r_{4}}=V_{r_{4}}$, it follows that $\nabla_{\xi} S=0$ is equivalent to $h-r_{1}-r_{2}=0$. Then we have the equation $(n-2) x^{4}-2 n x^{2}+n-2=0$. Hence, we find $x^{2}=(n \pm 2 \sqrt{n-1}) /(n-2)$ so that $x=(\sqrt{n-1}+1) / \sqrt{n-2}$, since $x>1$. Hence, $M$ is of case (3) in Theorem. Let $M$ be of type (D) (which is a tube of radius $r$ ). Let $x=\cot r$. Then $M$ has five distinct constant principal curvatures $r_{1}=(1+x) /(1-x)$ with multiplicity $4, r_{2}=(x-1) /(x+1)$ with multiplicity $4, r_{3}=x$ with multiplicity $4, r_{4}=-1 / x$ with multiplicity 4 , and $\alpha=\left(x^{2}-1\right) / x$ with multiplicity 1 (cf. [10]). By virtue of the discussion in case of type (C) we have only to solve the equation $h-r_{1}-r_{2}=0$. Namely, we get the equation $5 x^{4}-22 x^{2}+5=0$ so that $x=(\sqrt{8}+\sqrt{3}) / \sqrt{5}$. Hence $M$ is of case (4) in Theorem. Finally let $M$ be of type (E) (which is a tube of radius $r$ ). Let $x=\cot r$. Then $M$ has five distinct constant principal curvatures $r_{1}=(1+x) /(1-x)$ with multiplicity $6, r_{2}=(x-1) /(x+1)$ with multiplicity 6 , $r_{3}=x$ with multiplicity $8, r_{4}=-1 / x$ with multiplicity 8 , and $\alpha=\left(x^{2}-1\right) / x$ with multiplicity 1 (cf. [10]). Considering the equation $h-r_{1}-r_{2}=0$, we have the equation $9 x^{4}-38 x^{2}+9=0$ so that $x=(\sqrt{5}+\sqrt{14}) / 3$. Hence, $M$ is of case (5) in Theorem. Q.E.D.

Remarks. (1) The condition "the Kaehler submanifold $\tilde{N}$ does not have principal curvatures \pm 1 " in case (6) of Theorem and the condition "the Kaehler submanifold $\tilde{N}$ does not have principal curvatures $\pm((2 k-1) /(2 n-2 k-1))^{1 / 2}$ " in case (7) of Theorem are necessary. In general, Proposition C (ii) shows that 
the point $x \quad(=\gamma(0))$ is a singular point of $M$ (that is, $M$ is not smooth at the point $x$ ), when $r=\theta$.

(2) Case (2) in Theorem is the only tube over $Q_{n-1}$ which is pseudo-Einstein (cf. [1]). $2 k$.

(3) Case (7) in Theorem is nothing else but case (6) in Theorem, when $n=$

(4) The author does not know examples of manifolds $\tilde{N}$ having the properties required in Case (7) in Theorem in the case of $k \geq 2$. The following $\tilde{N}$ is an example in the case of $k=1$.

Let $\tilde{N}=P_{1}(C)$ (of holomorphic sectional curvature $4 / n$ ) be a complex curve imbedded into $P_{n}(C)$ (of holomorphic sectional curvature 4) through the Veronese imbedding of degree $n(\geq 3)$ (see [7, Remark, p. 83]).

Therefore, the tube of radius $\cot ^{-1}\left\{(1 /(2 n-3))^{1 / 2}\right\}$ over the complex curve $\tilde{N}$ must be an example of Case (7) in Theorem in the case of $k=1$ (see [7, p. 90-91]).

(5) Y. J. Suh considered another condition on the derivative of $S$. He showed the following (cf. [8]): Let $M$ be a real hypersurface of $P_{n}(C)$. Then $\xi$ is a principal curvature vector and $g\left(\left(\nabla_{X} S\right) Y, Z\right)=0$ for any $X, Y$, and $Z$ which are orthogonal to $\xi$ if and only if $M$ is locally congruent to one of the homogeneous real hypersurfaces of types $\left(\mathrm{A}_{1}\right),\left(\mathrm{A}_{2}\right)$, and $(\mathrm{B})$.

\section{ACKNOWLEDGMENT}

The author heartily thanks Professors Hisao Nakagawa, Patrick J. Ryan, and Makoto Kimura for their kind advice during the preparation of this paper. He also greatly appreciates the referee's valuable suggestions.

\section{REFERENCES}

1. T. E. Cecil and P. J. Ryan, Focal sets and real hypersurfaces in complex projective space, Trans. Amer. Math. Soc. 269 (1982), 481-499.

2. U. H. Ki, H. Nakagawa, and Y. J. Suh, Real hypersurfaces with harmonic Weyl tensor of a complex space form, Hiroshima Math. J. 20 (1990), 93-102.

3. M. Kimura, Real hypersurfaces in complex projective space, Trans. Amer. Math. Soc. 296 (1986), 137-149.

4. M. Kimura and S. Maeda, On real hypersurfaces of a complex projective space II, Tsukuba J. Math. 15 (1991), 547-561.

5. M. Kon, Pseudo-Einstein real hypersurfaces in complex space form, J. Differential Geom. 14 (1979), 339-354.

6. Y. Maeda, On real hypersurfaces of a complex projective space, J. Math. Soc. Japan 28 (1976), 529-540.

7. K. Ogiue, Differential geometry of Kaehler submanifolds; Adv. Math. 13 (1974), 73-114.

8. Y. J. Suh, On real hypersurfaces of a complex space form with $\eta$-parallel Ricci tensor, Tsukuba J. Math. 14 (1990), 27-37.

9. R. Takagi, On homogeneous real hypersurfaces in a complex projective space, Osaka J. Math. 10 (1973), 495-506.

10. _ـ Real hypersurfaces in a complex projective space with constant principal curvatures. I, II, J. Math. Soc. Japan 27 (1975), 43-53, 507-516.

Department of Mathematics, Nagoya Institute of Technology, Gokiso, Shôwa, NAGOYA, 466, JAPAN 